\title{
Seeing textons in context
}

\author{
JAMES ENNS \\ Dalhousie University, Halifax, Nova Scotia, Canada
}

\begin{abstract}
Texton theory holds that visual texture segregation occurs through the preattentive detection of local differences in primitive visual units called "textons." Three textons have been proposed for shape: lines, line terminators, and line intersections. The experiments reported here show that line closure also behaves like a texton under some conditions. In addition, the experiments show that texture segregation is not determined by texton differences per se, but by the extent to which the unique textons in a region are salient in the context of the textons common to regions. This suggests that preattentive vision is not as simple minded as texton theory claims it to be.
\end{abstract}

Perception researchers have long been intrigued by the ability of the human visual system to detect subtle differences in visual texture almost instantaneously over a large visual field (Mach, 1959; Wertheimer, 1950). Recent investigations have been aimed at identifying the fundamental visual features that permit texture segregation to occur (Beck, 1974, 1983; Bergen \& Julesz, 1983; Julesz, 1980, 1981; Julesz \& Bergen, 1983; Pomerantz, 1981; Treisman \& Gelade, 1980; Treisman \& Paterson, 1984). The most complete model of texture segregation is Julesz's "texton theory"' (e.g., Julesz \& Bergen, 1983). This theory makes two strong claims that are called into question by the experiments reported here.

The first claim is that the human visual system consists of two distinct systems: a preattentive system that operates instantaneously, in parallel, over a large visual field (at least $14^{\circ}$ of arc) and an attentive system that operates by moving a small "spotlight" serially over a visual array in 50-msec steps. ${ }^{1}$ The second claim is that there are a small, fixed number of textons that can be detected by the preattentive system. The three textons that have been proposed for shape are: elongated blobs, or lines, of specific colors, widths, lengths, and orientations, terminations of elongated blobs, or line terminators; and blob crossings, or line intersections. The preattentive system is limited to detecting local differences in the type and number of these textons anywhere in the visual field. It then directs attention to those regions for closer inspection. Other features defined by the positional relationships between textons (e.g., line closure) are ignored by the preattentive system and can be detected only with attentional scrutiny. ${ }^{2}$

These claims can be perceived intuitively by inspection of the textures illustrated in Figure 1. Figure 1A is com-

This research was funded by a grant from the Natural Sciences and Engineering Research Council of Canada. I am grateful to Bill Prinzmetal, Ray Klein, John Barresi, Bruce Earhard, and Anne Treisman for helpful criticism. Aaron Gilani deserves special thanks for running the subjects in Experiments 1 and 2. Reprint requests may be addressed to James Enns, Department of Psychology, Dalhousie University, Halifax, Nova Scotia, Canada B3H 4J1.
A
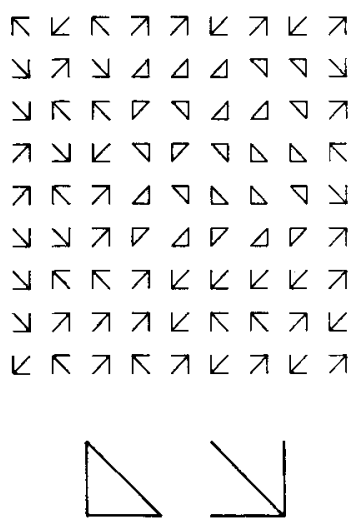

Figure 1. Two textures that support texton theory. According to this theory, the region in the upper right of texture $A$ is segregated from the background because the elements differ in number of line terminators (3:0). The same region in texture $B$ does not segregate because the two elements have the same number of line terminators (2:2).

posed of two elements: a triangular element and an arrowlike element. The two elements, shown below the texture, both contain one vertical line, one horizontal line and one diagonal line. They differ only in the location of the vertical line. The texture was created by placing an element on each intersection of an imaginary $9 \times 9$ grid, in one of two orientations and in one of two reflections, on a random basis. As can be seen, the region containing the triangles is visually segregated from the background which contains only arrows. Because the elements have the same lines, the strong segregation cannot be the result of differences in number or type of lines. One candidate visual property responsible for the segregation is line closure. This property characterizes the triangle but not the arrow. A second candidate is the difference in the number of line terminators. The triangle has no terminators, whereas the arrow has three. Julesz prefers the latter interpretation on the basis of Figure 1B.

Figure 1B is also composed of two elements that differ in the location of a single line: an " $S$ "-like element and 
an element resembling a supine " 10. ." In addition to sharing three horizontal lines and two vertical lines, these elements each have two line terminators. Like Figure 1A, the two elements differ in that one has line closure and the other does not. In contrast to Figure $1 \mathrm{~A}$, the region containing the " $10 \mathrm{~s}$ " is very difficult to distinguish even though it appears in the same location as the triangles. Thus, Figure 1B is used by Julesz to argue that line closure cannot be detected preattentively when the number of line terminators is held constant.

One potentially important aspect of texture segregation that is ignored by texton theory is the relative salience of the unique features. Texton theory predicts texture segregation on the basis of a simple difference in texton type or number. An alternative idea, expressed originally by Olson and Attneave (1970) and more recently by Beck (1983), suggests that texture segregation may occur to the extent that the variation (differences) in features between regions is greater than the variation in features within a region. Hence, texture segregation may not be determined by texton differences per se, but by the extent to which the unique textons in elements are salient in the context of the textons common to elements.

If Figure 1 is reconsidered from this point of view, an alternative explanation for the differential segregation of these two textures becomes apparent. Both textures are composed of elements that differ only in the location of a single vertical line. However, the unique line in Figure 1A is similar in length to the common horizontal lines, whereas the unique line in Figure $1 \mathrm{~B}$ is only $1 / 5$ the length of the common horizontal lines. The present study asked whether texture segregation is influenced by such differences in the relative salience of the unique element features.

\section{EXPERIMENT 1}

\section{Method}

The textures in Figure 1 were modified as shown in Figure 2. The triangles and arrows were changed so that the unique vertical line was $1 / 5$ the length of the common horizontal line. Conversely, the " $S$ " and " 10 " elements were changed to make the unique vertical line the same length as the common horizontal lines. For convenience, shorthand will be used to refer to the four texture types in Figures 1 and 2. Triangles and arrows with unique vertical line equal to common horizontal line will be referred to as TA-1 (Figure 1A); "Ss" and " $10 \mathrm{~s}$ " with unique vertical line 1/5 the length of horizontal lines, ST-0.2 (Figure 1B); triangle-arrow with unique line $1 / 5$ the common line, TA-0.2 (Figure $2 \mathrm{~A}$ ); and " $\mathrm{S}-10$ " with unique line equal to common lines, ST-1 (Figure 2B).

The base and sides of the TA-1 elements subtended $0.32^{\circ}$ of arc, as seen by the subjects. The long segments of the ST- 0.2 elements subtended $0.64^{\circ}$, the short segments $0.13^{\circ}$; the long side of the TA-0.2 elements subtended $0.80^{\circ}$, the short side $0.16^{\circ}$; and all segments in the ST-1 elements subtended $0.26^{\circ}$ of arc. The center-tocenter distance between elements in the textures was $0.93^{\circ}$ of arc. Thus, the $9 \times 9$ element texture subtended approximately $8^{\circ}$ of arc. The odd region of a texture was a $5 \times 5$ element region in one of the quadrants. This region overlapped the center of the display field by $1 \frac{1}{2}$ rows and $11 / 2$ columns of elements $\left(1.4^{\circ}\right.$ of arc) to ensure that texture boundaries would not fall on the fovea except by chance. The luminance of the textures was $2.8-3.2 \mathrm{~cd} / \mathrm{m}^{2}$.

A total of 64 different textured stimuli were generated: 32 con-
A

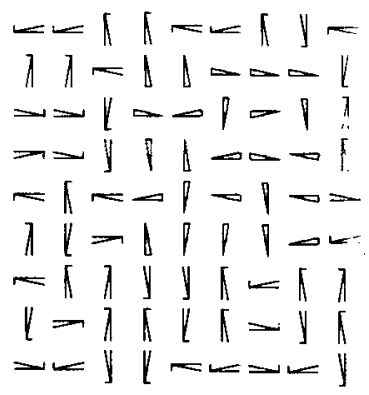

$=-\|\|=-\|\|=$

$11-1 \mid=-\infty$

$=-\|<-1-1 ?$

$=1-1 \rightarrow 1 \rightarrow$

$11=18+1-\infty$

$-\pi 1$ I $\|-\| 1$

$=-\|\|=-\infty)$

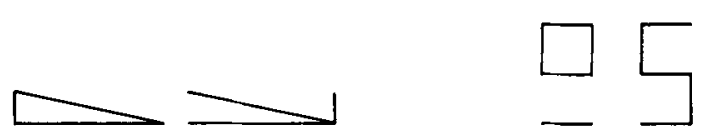

Figure 2. Two textures that violate texton theory. These textures contain the same texton differences as Figure 1, but the relative degree of segregation has been reversed. This was achieved by simply shortening the unique line in $A$ and increasing the length of the unique line in $B$.

tained the same elements (homogeneous) and 32 contained an odd region in one of the quadrants (heterogeneous). The homogeneous set consisted of four different textures for each of the two elements involved in each of the four texture types. The heterogeneous set contained four textures (one for each possible odd quadrant) for each of the two figure-ground possibilities (e.g. , triangles on a background of arrows, arrows on a background of triangles) for each of the four texture types.

Each subject was presented with a total of 512 trials in one session lasting $1 \mathrm{~h}$. The trials were divided into eight blocks, and within each block the 64 stimuli were presented in a random order. On each trial, a high-contrast texture was presented for $150 \mathrm{msec}$, preceded and followed by a field of equal luminance containing a central fixation point. The subject said "yes" or "no" after each presentation to indicate whether the texture contained an odd region, and was instructed to guess when uncertain. A Gerbrands threefield tachistoscope was used to present the stimuli. Nine adults (8 university students, 1 faculty member) with normal or correctedto-normal vision participated as subjects.

\section{Results}

The percentage of correct responses for each texture type and subject are presented in Table 1. As predicted by texton theory, TA-1 textures were segregated significantly better than the chance level of $50 \%[t(8)=29.91$, $p<.01$ ], whereas ST-0.2 textures were not discriminated

Table 1

Percent Correct in the Detection Task in Experiment 1

\begin{tabular}{ccccc}
\hline & \multicolumn{5}{c}{ Texture Type } \\
\cline { 2 - 5 } Subject & TA-1 & ST-0.2 & TA-0.2 & ST-1 \\
\hline 1 & 98 & 52 & 73 & 96 \\
2 & 100 & 55 & 84 & 96 \\
3 & 95 & 41 & 68 & 79 \\
4 & 94 & 50 & 53 & 88 \\
5 & 98 & 45 & 80 & 94 \\
6 & 99 & 50 & 62 & 90 \\
7 & 93 & 46 & 73 & 85 \\
8 & 97 & 49 & 82 & 88 \\
9 & 85 & 46 & 55 & 73 \\
Mean & 95 & 48 & 70 & 88 \\
\hline
\end{tabular}

Note-Chance accuracy is $50 \%$. 
significantly better than chance $[t(8)=1.28]$. However, contrary to texton theory, ST-1 textures were also discriminated significantly better than chance $[t(8)=14.56$, $p<.01]$. The TA- 0.2 textures were discriminated significantly better than chance $[t(8)=5.26, p<.01]$ but more poorly than both TA-1 textures $[t(8)=8.27$, $p<.01]$ and ST-1 textures $[t(8)=5.69, p<.01]$.

\section{EXPERIMENT 2}

A second experiment generalized the results from the presence-absence detection task to a quadrant location task similar to the one used by Julesz (1980). This time only the 32 textures with odd regions were used and the subjects' task was to locate the odd quadrant. Four adult subjects were each run on 320 trials divided into 10 blocks; otherwise the procedure and stimuli were identical to those of Experiment 1. Table 2 shows that the pattern of results is also similar. The odd regions were located in TA-1 textures with accuracy well above the chance level of $25 \%$ $[t(3)=12.47, p<.01]$ but not significantly better than chance in ST-0.2 textures $[t(3)=1.71]$. However, the subjects were again able to locate the odd region in ST-1 textures with better than chance accuracy $[\mathrm{t}(3)=11.08$, $p<.01]$ and were unable to do so with the TA-0.2 textures $[t(3)=1.36]$. The subjects were also less able to locate the odd region in TA-0.2 textures than in TA-1 $[t(3)=10.07, p<.01]$ or in ST-1 textures $[t(3)=14.89$, $p<.01]$.

\section{EXPERIMENT 3}

In a final experiment, the textures were modified to address two potential critcisms. First, differences in segregation between textures with short unique lines (TA-0.2, ST-0.2) and long unique lines (TA-1, ST-1) may be a function of poor acuity for the short lines, rather than a function of the relative lengths of the unique versus common lines. To test this idea, the absolute sizes of the texture elements were doubled so that the unique lines in TA-0.2 and ST- 0.2 were the same length as the unique lines of TA-1 and ST-1 in Experiments 1 and 2. Since this manipulation enlarged the texture elements and the distance between elements proportionally, the textures now consisted of a $5 \times 5$ grid of elements with a $3 \times 3$ odd quadrant in one of the corners.

The second criticism was that the superior segregation of TA-1 over other textures might be confounded by a

Table 2

Percent Correct in the Location Task in Experiment 2 Texture Type

\begin{tabular}{ccccc}
\hline & \multicolumn{4}{c}{ Texture Type } \\
\cline { 2 - 5 } Subject & TA-1 & ST-0.2 & TA-0.2 & ST-1 \\
\hline 1 & 64 & 29 & 31 & 60 \\
2 & 76 & 30 & 26 & 56 \\
3 & 67 & 25 & 24 & 50 \\
4 & 80 & 25 & 27 & 49 \\
Mean & 72 & 27 & 27 & 54 \\
\hline
\end{tabular}

Note-Chance accuracy is $25 \%$.
Table 3

Percent Correct in the Location Task in Experiment 3

\begin{tabular}{ccccc}
\hline & \multicolumn{4}{c}{ Texture Type } \\
\cline { 2 - 5 } Subject & TA-1 & ST-0.2 & TA-0.2 & ST-1 \\
\hline 1 & 84 & 25 & 46 & 77 \\
2 & 66 & 23 & 46 & 56 \\
3 & 96 & 23 & 64 & 79 \\
4 & 89 & 30 & 47 & 63 \\
Mean & 84 & 25 & 51 & 69 \\
\hline
\end{tabular}

Note-Chance accuracy is $25 \%$.

unique property of that texture. The vertices and edges of the elements are neatly aligned in TA-1 textures, whereas there is no similar alignment in any of the other textures. In Experiment 3, elements in all the textures were oriented at an angle chosen randomly between $0^{\circ}$ and $360^{\circ}$ to eliminate this difference.

Four adult subjects were again run on 320 trials, following the same procedure as in Experiment 2. Table 3 shows that the pattern of results did not change. TA- 1 and ST-1 textures were discriminated above the chance level of $25 \%[t(3)=9.17, p<.01$, and $t(3)=7.89, p<.01$, respectively], whereas the ST- 0.2 textures were not $[t(3)$ $=0.15]$. Subjects located the odd region more accurately in both TA- 1 and ST- 1 textures than in TA-0.2 textures $[t(3)=6.88, p<.01$, and $t(3)=3.98, p<.05$, respectively], and the TA-0.2 textures were discriminated at an above-chance level $[t(3)=5.82, p<.05]$.

\section{DISCUSSION}

Two main findings of the present experiments are not easily accommodated by texton theory in its present form. The first finding is that the ST-1 textures were discriminable preattentively even though they contained no differences in textons that have been identified by Julesz. In fact, these textures were distinguished more easily than the TA- 0.2 textures, which did contain differences in textons (a 3:0 difference in number of line terminators). One way to resolve this discrepancy might be to design more stringent criteria for features to pass before being called textons. However, this strategy would almost certainly lead to the failure of some "known" textons under some conditions. A present case in point is the failure of terminator differences of 3:0 to permit segregation of the TA0.2 textures in Experiment 2. Another way to resolve this discrepancy might be to simply expand the texton alphabet to include line closure (see Treisman \& Paterson, 1984, for additional support for the notion of closure as a visual primitive). However, because closure seems to act like a texton under some conditions but not under others, following this strategy would change the notion of texton from that of a categorically defined feature to that of a "graded" feature, that is, one that can be present in varying degrees (Treisman \& Souther, 1985). Furthermore, if the notion of "texton" is extended to include both categorical and graded features, its theoretical power as a term for a small, unique set of visual properties is diluted. 


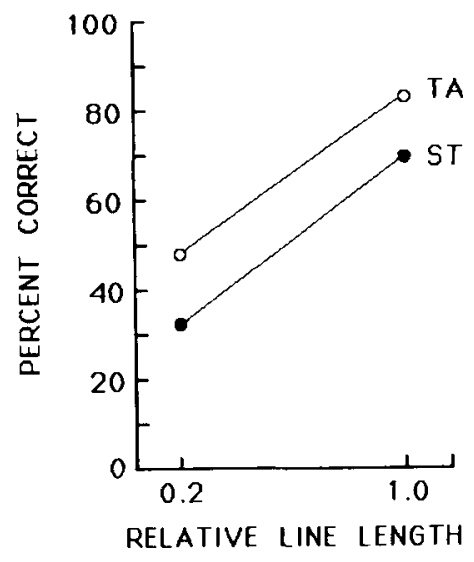

Figure 3. Mean percent correct combined over the three experiments. The data have been plotted as a function of unique line length (0.2 vs. 1.0) and type of texture (TA vs. ST), in order to show the relative contribution of each factor to texture segregation.

The second and larger problem for texton theory is the apparent context dependency or "graded" nature (Treisman \& Souther, 1985) of all the textons examined in the present study. In each experiment, discrimination accuracy was affected more by the relative length of the unique element line than by differences in texton type or number. This point is made quite clearly in Figure 3. Here the accuracy data have been pooled over the three experiments and plotted as a function of two factors: the relative length of the unique line in the texture elements $(0.2$ vs. 1) and the presence of possible texton differences (closure in the ST textures vs. closure and line terminators in the TA textures). There is a striking difference in the relative magnitude of effect that these two factors have on discrimination accuracy. The TA textures are discriminated approximately $15 \%$ more accurately than the ST textures, presumably because of the additional texton differences in TA textures. However, this difference appears to be additive with the much larger improvement in accuracy $(36 \%)$ that occurs as a function of unique line length. This result strikes directly at the claim that local texton differences are detected in an all or none fashion, and thus that textons are fundamental units in preattentive vision. Instead, these results support Beck's (1983) notion that texture regions are segregated through the computation of a similarity function that takes into account both common and unique features in a local area. The context dependency of textons also raises questions about the strong dichotomy proposed by texton theory to distinguish preattentive and attentive vision. Rather than pointing to two visual systems that are qualitatively distinct, texture segregation experiments may be tapping a continuum of discrimination difficulty (i.e, acuity) in the retinal periphery, as originally suggested by Beck (1974).

Treisman and Souther (1985) recently provided several other demonstrations which showed that line closure may or may not be detected categorically, depending on the nature of the background elements. Using a visual search paradigm and response time (RT) as the primary dependent measure. Treisman and Souther (1985, Experiment 3 ) found that search for a triangle against a background of lines and angles (components of the triangles) yielded shallow RT functions ( $<5 \mathrm{msec}$ per additional distractor element). Similarly, search for a circle against a background of semicircles resulted in a relatively flat RT function $(<10 \mathrm{msec}$ per distractor). These authors interpreted such flat RT functions to indicate parallel (preattentive) detection of the circle targets. However, they also showed that search for circle targets in the context of circles with intersecting lines (Experiments 1 and 2) and search for circles in the context of circles with gaps (Experiment 4) resulted in steep RT functions (20-60 msec per distractor). That is, in these latter contexts, search for circles was serial (with attention). Treisman and Souther accounted for the unruly behavior of the closure property in these experiments by suggesting that the definition of "closure"' as a categorical property (the absence of line terminators) was inappropriate. Instead, they suggested that closure be defined as a graded property: the extent to which an area is enclosed by a convex contour. In their view, when closure is a property of both target and distractor elements, the speed of the serial search for the target will be determined by the extent to which the amount of closure is similar in target and distractor elements. Only when closure is completely absent from the background will a target with closure be detected preattentively. Thus, this account suggests that the ST- 0.2 textures are less discriminable than the ST-1 textures because the former textures are more similar in terms of their total convexity. Whether one prefers the Treisman and Souther interpretation that is based on shared convexity or the one given above that is based on the salience of the unique feature (line) relative to the salience of the common features (closure, common lines), the present results agree with those of Treisman and Souther in suggesting that closure is not a feature that is coded categorically in preattentive vision.

Unlike the present findings, Treisman and Souther (1985) did not find line terminators to be context dependent. They reported that search for targets containing line terminators against a background of elements without line terminators was affected very little by the number of distractors $(<5 \mathrm{msec}$ per additional distractor in Experiments 1,2 , and 4 ) and not at all by the similarity of the target and background elements (Experiment 4). However, it is difficult to make direct comparisons between Treisman and Souther's experiments and the present study because of the different assumptions that were involved in the construction of stimuli. Following Julesz (1980), the elements within a given texture in the present study were designed to vary in the type and number of proposed textons while lower order features such as the total line length and line orientation were held constant. The elements used in Treisman and Souther's search experiments always differed in at least one way in addition to the feature of interest. For example, targets consisting 
of circles with intersecting lines were placed among distractors consisting of circles alone. Texture regions composed of these two elements are guaranteed to segregate simply on the basis of differences in overall density (see Julesz, 1980, 1981). Thus, a complete understanding of the differences between the present data and those of Treisman and Souther (1985) would require a systematic comparison of the differences in both procedure and stimuli in the two studies. In any event, the variation in discriminability between the TA-1 and TA- 0.2 textures in the present experiment can be seen as an important first demonstration that line terminators also behave as graded features rather than as categorical ones.

Tversky's (1977; Tversky \& Gati, 1978) contrast model of similarity provides a convenient framework within which to consider both Julesz's view and Beck's view of texture segregation. In its more general form, the contrast model holds that the similarity between objects (texture regions in this case) is a function of both the features common to the two objects and the features unique to the two objects. It also predicts that the consequence of increasing the salience of common features will be to decrease the salience of unique features, and vice versa. This general model is consistent with Beck (1983), provided that one is willing to assume that discriminability is an inverse measure of similarity, that the features are textons such as lines, intersections, terminators, and closure, and that one way in which the salience of a line can be strengthened is by increasing its relative length. However, there is also a special case of the contrast model in which similarity is a function only of differences in the number or type of unique features. Common features are simply ignored. This special case of the contrast model seems to characterize the position of Julesz (1980; Julesz \& Bergen, 1983). The present results clearly favor the general contrast model over the special model as a description of the segregation of visual textures on the basis of form.

\section{REFERENCES}

BECK, J. (1974). Relation between similarity grouping and peripheral discriminability. Journal of Experimental Psychology, 102, 1145-1147. BECK, J. (1983). Textural segmentation. In J. Beck (Ed.), Organization and representation in perception. Hillsdale, NJ: Erlbaum.
Bergen, J. R., \& JULesz, B. (1983). Parallel versus serial processing in rapid pattern discrimination. Nature, 303, 696-698.

JAMES W. (1890). The principles of psychology (Vol. 1). New York: Dover.

JULESZ, B. (1980). Spatial nonlinearities in the instantaneous perception of textures with identical power spectra. Philosophical Transactions of the Royal Society of London B, 290, 83-94.

Julesz, B. (1981). Textons, the elements of texture perception, and their interactions. Nature, 290, 91-97.

Julesz, B., \& BERGEN, J. R. (1983). Textons, the fundamental elements in preattentive vision and perception of textures. Bell System Technical Journal, 62, 1619-1645.

MaCH, E. (1959). The analysis of sensations. New York: Dover.

Neisser, U. (1967). Cognitive psychology. Englewood Cliffs, NJ: Prentice-Hall.

Olson, R., \& AtTNEAVE, F. (1970). What variables produce similarity grouping? American Journal of Psychology, 83, 1-21.

Pomerantz, J. R. (1981). Perceptual organization in information processing. In M. Kubovy \& J. R. Pomerantz (Eds.), Perceptual organization. Hillsdale, NJ: Erlbaum.

Treisman, A., \& Gelade, G. (1980). A feature integration theory of attention. Cognitive Psychology, 12, 97-136.

Treisman, A., \& Paterson, R. (1984). Emergent features, attention and object perception. Journal of Experimental Psychology: Human Perception \& Performance, 10, 12-31.

Treisman, A., \& Souther, J. (1985). Search asymmetry: A diagnostic for preattentive processing of separable features. Journal of $E x$ perimental Pscyhology: General, 114, 285-310.

TVERSKY, A. (1977). Features of similarity. Psychological Review, 84, 327-352.

Tyersky, A., \& Gati, I. (1978). Studies of similarity. In E. Rosch \& B. B. Lloyd (Eds.), Cognition and categorization. Hillsdale, NJ: Erlbaum.

WERTHEIMER, M. (1950). Laws of organization in perceptual forms. In W. D. Ellis (Ed.), A source book of Gestalt psychology. London: Routledge \& Kegan Paul. (Original work published 1923)

\section{NOTES}

1. The general distinction between preattentive and attentive vision has been made by others, including James (1890) and Neisser (1967). The specific claim for the speed of serial search was made by Julesz (1980).

2. Beck (1983) presents results that support the same claim, and Treisman and Paterson (1984) present results from visual search, texture segmentation, and illusory conjunction experiments that implicate line closure as a fundamental feature.

(Manuscript received October 4, 1985; revision accepted for publication February 17, 1986.) 